\title{
Small-angle lattice rotations in graphene on $\mathrm{Ru}(0001)$
}

\author{
K. L. Man and M. S. Altman* \\ Department of Physics, Hong Kong University of Science and Technology, Clear Water Bay, Kowloon, Hong Kong
}

(Received 25 July 2011; published 1 December 2011)

\begin{abstract}
Lattice rotations in graphene on $\mathrm{Ru}(0001)$ are investigated with low-energy electron microscopy and microlow-energy electron diffraction. The measurements place an upper limit, $250 \mathrm{~nm}$, on the racemic length scale of the recently reported chirality in this system, arising from rotated features within the unit cell. On a longer length scale, small rotations from orientational coincidence with the substrate lattice are found to be present in the vast majority of the graphene layer. The resulting proliferation of small-angle grain boundaries may influence the properties of the graphene layer that is otherwise free of large-angle rotational variants and oriented uniformly with the substrate.
\end{abstract}

DOI: 10.1103/PhysRevB.84.235415

PACS number(s): 61.48.Gh, 61.05.jh, 68.35.Dv, 68.37.Nq

\section{INTRODUCTION}

Wide interest in graphene on metal surfaces is partly motivated by the necessity to make metallic contacts in graphene-based devices. It is also believed that growth on metals offers the advantage of producing high-quality singlelayer graphene over large areas. ${ }^{1-5}$ Meanwhile, it is widely recognized that the novel electronic structure and properties of a graphene layer that have attracted such wide interest ${ }^{6}$ can be influenced by its interaction with a supporting metal substrate. ${ }^{7-13}$ This interaction differs among the metals and can even differ among the various large-angle rotational variants that occur on the same metal surface. ${ }^{12,13}$ The formation of such rotational variants on many metal surfaces ${ }^{4,9,12-17}$ also introduces grain boundaries into the graphene layer. These defect structures are believed to affect, and therefore may offer a means for manipulating electronic and transport properties. $^{17-22}$

Among the numerous metals that support the growth of graphene, the morphology and structure of graphene on the $\mathrm{Ru}(0001)$ surface are particularly interesting. Unlike its morphology on most other metals, graphene grows with essentially one orientational relationship on $\mathrm{Ru}(0001)$, with coincident high-symmetry directions. ${ }^{1,3,11,23}$ Several aspects of the structure of graphene $(\mathrm{g})$ on the $\mathrm{Ru}(0001)$ surface have also stirred debate. With superior reciprocal space resolution, surface $x$-ray diffraction (SXRD) measurements conclusively demonstrated the superposition of $(25 \times 25) \mathrm{g}$ on $(23 \times$ 23) $\mathrm{Ru}$ units $^{24}$ and ruled out 12-on-11 (Refs. 3, 23, 25, and 26) and 11-on-10 (Ref. 27) periodicities that have also been discussed. Substantial corrugation was also observed with scanning tunneling microscopy (STM) $)^{3,23,24,26,27}$ that has been alternatively interpreted in terms of predominantly physical buckling ${ }^{3,24}$ or electronic effects, ${ }^{26,27}$ with the former supported by SXRD, ${ }^{24,28}$ low-energy electron diffraction (LEED), ${ }^{29}$ density functional theory calculations, ${ }^{3,29}$ and $\mathrm{Xe}$ spectral features and desorption, ${ }^{30}$ and the latter by helium atom scattering. ${ }^{26}$ Large corrugated features are coupled with the moiré pattern that is produced by the superposition of the mismatched $\mathrm{g}$ and $\mathrm{Ru}$ lattices. These feature are present in a $(2 \times 2)$ arrangement within the 25 -on- 23 cell. The origin of the surprisingly large unit cell comprising multiple rather than a single moiré feature is still not understood.
Intriguing evidence from SXRD was also put forth that $\mathrm{g} / \mathrm{Ru}(0001)$ is chiral, whereby each of the weakly bound, protruding regions within a physically corrugated unit cell is rotated in-plane in the same direction by up to $2^{\circ} .^{28}$ This structure was rationalized by calculations that demonstrated the elastic energy due to strain to be substantially lower for the chiral graphene structure than for the best-fit nonchiral corrugated structure. ${ }^{28}$ Consistent with this argument, it was also noted that bond lengths in chiral graphene are more preserved relative to bulk graphite than in the nonchiral structure. $^{28}$

The formation of moiré-like superstructures on many supporting crystalline substrates that give rise to corrugation is known to facilitate the function of supported graphene for atomic, molecular, or metallic cluster adsorption. ${ }^{9,30-32}$ Chirality may enhance this functionality, e.g., for molecular chiral recognition. ${ }^{28}$ The presence of physical corrugation in systems that form a moiré superposition has been attributed to, and contributes to, the spatially varying proximity of carbon and metal atoms and the strength of their interaction within the unit cell. Lateral variations of electronic properties within the unit cell have also been correlated with the spatially varying bonding strength between graphene and metal substrate.9,26,27 The symmetry breaking that is associated with chirality in the protruding regions of a physically corrugated graphene layer may contribute to these variations, possibly even inducing spin polarization effects. ${ }^{33,34}$ Differing amounts of charge transfer with the substrate in the strongly bound, low-lying and weakly bound, protruding regions ${ }^{9}$ should also produce local electric fields that could impact on these effects. ${ }^{34}$

Despite the compelling arguments for its existence and the intriguing potential implications, chirality has not yet been confirmed. It should be noted that two chiral enantiomers should be present, rotated clockwise and counterclockwise, respectively. Each enantiomer lowers the symmetry of the graphene lattice. Furthermore, due to the hcp bulk stacking sequence, the $\mathrm{Ru}(0001)$ surface may exhibit two terminations, each with threefold rotational symmetry and mutually rotated by $60^{\circ}$ about the surface normal. Given the large SXRD beam size, lateral averaging occurs over the four possible configurations of enantiomer and termination. Thus direct qualitative evidence of chirality in the symmetry of the diffraction peak intensities was not accessible to SXRD. 
Likewise, the characteristic length scale above which chiral $\mathrm{g} / \mathrm{Ru}(0001)$ is racemic is not known.

In this paper, we describe investigations that critically assess the existence of chirality in $\mathrm{g} / \mathrm{Ru}(0001)$ by addressing the limitation of SXRD to resolve signal spatially from a single configuration of chiral enantiomer and surface termination. Using low-energy electron microscopy (LEEM) and microLEED ( $\mu$ LEED), we have successfully obtained diffraction information from an area with a diameter of $250 \mathrm{~nm}$ within a single surface termination. While no evidence is found with such high-spatial-resolution diffraction measurements that confirms chiral features within the unit cell, we do in fact observe rotation of the entire graphene lattice on a much larger length scale. These observations revise our view of the orientational relationship in $\mathrm{g} / \mathrm{Ru}(0001)$ and may have broader implications for all other $\mathrm{g} / \mathrm{metal}$ systems. Evidence of a small biaxial lattice distortion that is coupled with the surface termination is also seen here in highly spatially resolved diffraction measurements. This distortion has not yet been considered in structure determinations of this intriguing system using laterally averaging techniques.

\section{RESULTS AND DISCUSSION}

\section{A. Experimental details}

Single-layer graphene was prepared by exposing a clean $\mathrm{Ru}(0001)$ surface to ethylene at fixed high temperature, which varied between 1110 and $1280 \mathrm{~K}$ in different experiments. Gas dosing in the pressure range of $1.5-3.5 \times 10^{-8}$ Torr produced a nearly complete layer within $1-2 \mathrm{~h}$. The individual graphene nuclei grew into very large flakes with dimensions of several tens of micrometers or larger under these conditions before coalescence. Subsequent LEEM and $\mu$ LEED measurements were carried out between 300 and $400 \mathrm{~K}$. In LEEM, a surface is imaged using elastically backscattered low-energy electrons. ${ }^{35,36}$ In $\mu$ LEED, diffraction information from a very small selected area is obtained by restricting the size of the electron beam that is incident on the sample. ${ }^{35-38}$ This is done in a LEEM instrument by placing a small aperture in the beam path. The beam size is further reduced by the demagnification $(\sim 20 \times)$ of the objective lens. An area with diameter of $250 \mathrm{~nm}$ was illuminated using a $5 \mu \mathrm{m}$ aperture in the present work. The size of the illuminating beam is confirmed directly in the LEEM image that is formed with the beam-limiting aperture in place. As discussed below, it is also confirmed independently in diffraction measurements. This illumination is much smaller than can be achieved in a conventional LEED apparatus.

\section{B. Chirality}

A $\mu$ LEED pattern of $g / R u(0001)$ is shown in Fig. 1. The different terminations of the $\mathrm{g} / \mathrm{Ru}(0001)$ surface are also evident in dark-field (DF) images formed using the $(\overline{2} / 23,2 / 23)$ and $(\overline{2} / 23,0)$ diffraction beams (Fig. 2). The brightness of a region in a DF image depends upon how strongly that region scatters to the diffraction peak used for imaging. Contrast in these images between adjacent terraces separated by a single atomic step identifies the spatial separation of the two terminations. Contrast reversal is observed between these two images because the two imaging beams are separated by
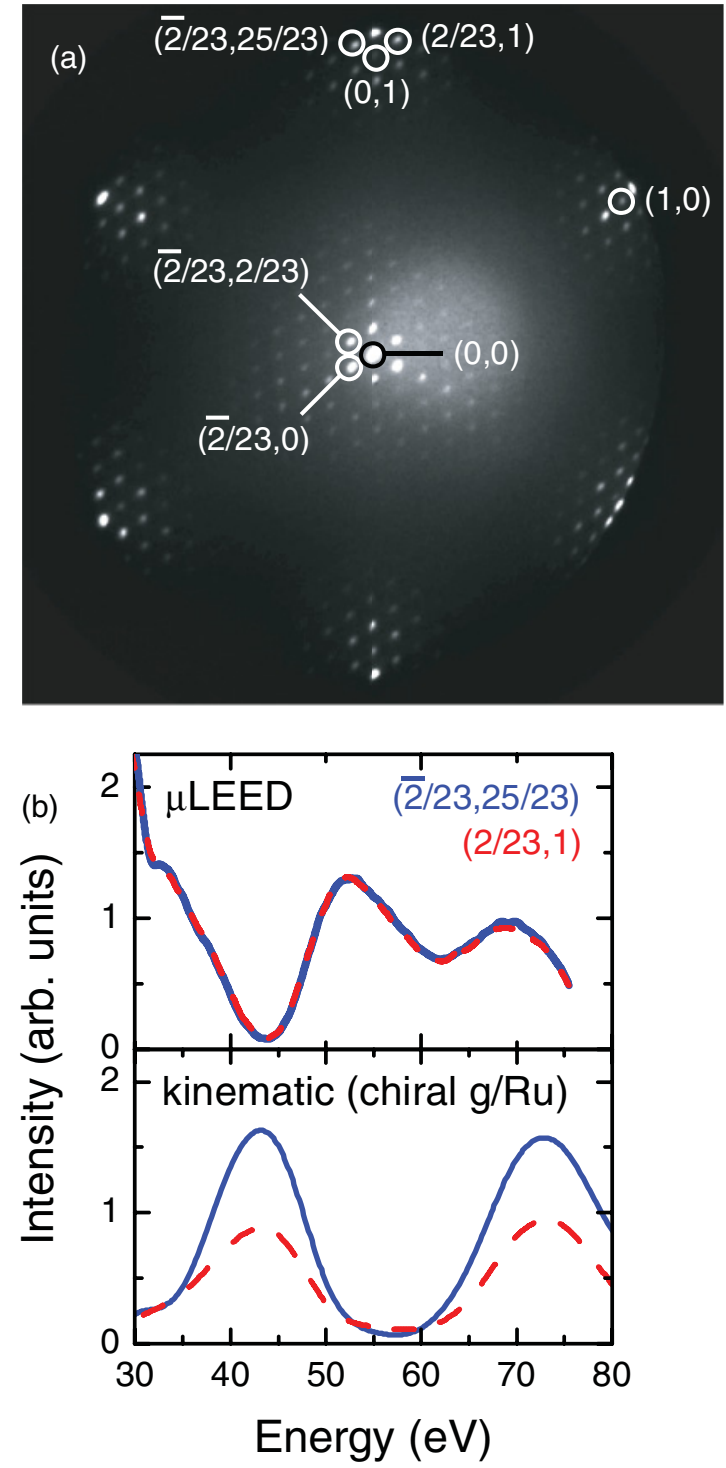

FIG. 1. (Color online) A $\mu$ LEED pattern of $g / R u(0001)$ at incident electron energy $E=31.5 \mathrm{eV}$ is shown in (a). (b) The intensity vs energy $I(V)$ curves for the $(\overline{2} / 23,25 / 23)$ (blue, solid curve) and $(2 / 23,1)$ (red, dashed curve) spots that are shown were (upper) measured experimentally with $\mu$ LEED for $\mathrm{g} / \mathrm{Ru}(0001)$ from a single surface termination and (lower) calculated kinematically for chiral g/Ru(0001).

$60^{\circ}$, half of the angular interval consistent with the threefold rotational symmetry of each termination. Similar contrast on the clean $\mathrm{Ru}(0001)$ surface was also observed previously in DF images formed using the integer order (10) and (01) diffraction spots. ${ }^{38}$

Measurements of diffraction intensities were made with the $\mu$ LEED beam positioned on a single terrace in order to search for evidence of chirality. If the system is nonchiral, then intensities of different diffraction peaks produced by a single termination should exhibit the $p 3 m 1$ symmetry of the graphene and $\mathrm{Ru}$ surface termination, namely, threefold rotational symmetry with mirror symmetry planes. On the other hand, chirality reduces symmetry to $p 3$. This should give rise to differences between the intensities of diffraction spots 


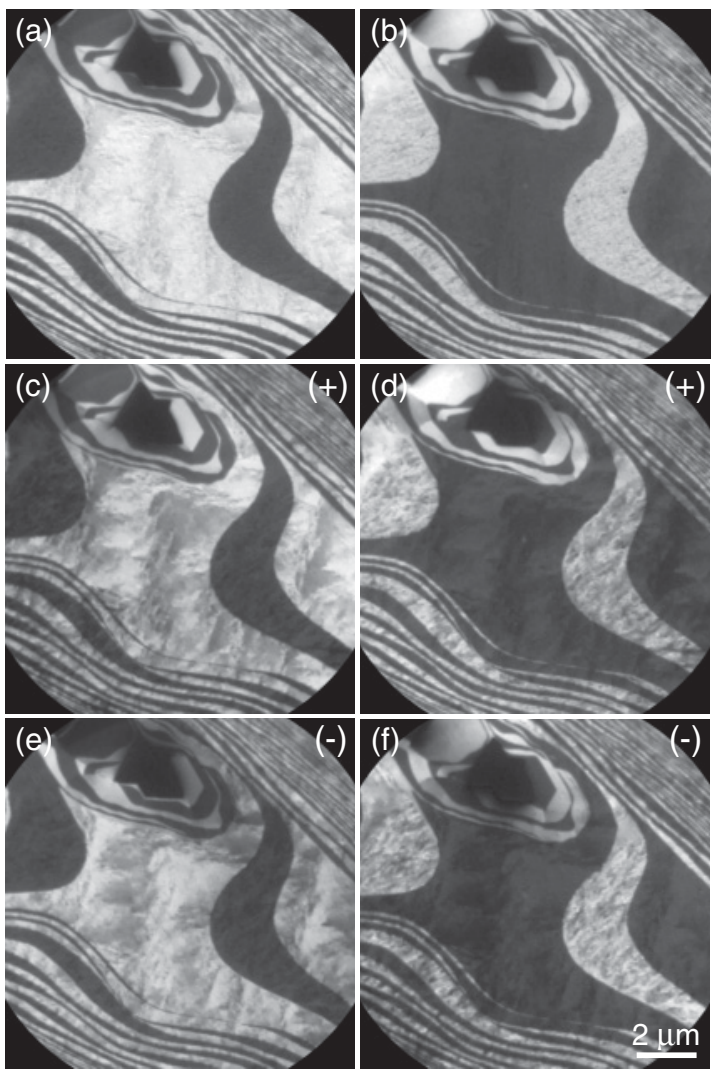

FIG. 2. (a),(b) Dark-field (DF) and (c)-(f) shadow dark-field (SDF) images of $\mathrm{g} / \mathrm{Ru}(0001)$ formed using the (a),(c),(e) $(\overline{2} / 23,2 / 23)$ and (b),(d),(f) $(\overline{2} / 23,0)$ diffraction spots are shown (imaging energy $E=10 \mathrm{eV}$ ). The labels (+) in (c),(d) and (-) in (e),(f) indicate SDF images formed by opposite relative displacements of the diffraction spot and contrast aperture. Contrast in (a),(b) identifies different terminations of the substrate on terraces separated by single atomic steps. Rotation domains in the graphene lattice are additionally evident in (c)-(f).

mirrored across the high-symmetry directions of the nonchiral surface. The broken mirror symmetry is seen, for example, in the diffraction intensities versus incident electron energy $I(V)$ that are calculated kinematically for the $(\overline{2} / 23,25 / 23)$ and $(2 / 23,1)$ spots using the structural parameters of chiral $\mathrm{g} / \mathrm{Ru}(0001)$ determined by SXRD [Fig. 1(b)]. ${ }^{28,39}$ The kinematic calculation demonstrates that the effect of chirality is significant and consequently, that it should be easily detectable with SXRD if a single chiral enantiomer on a single surface termination could be isolated. Note, incident energy in Fig. 1(b) is related to perpendicular momentum transfer.

In contrast, the measured $I(V)$ curves for the same two spots [Fig. 1(b)] are identical. Similar results for all other diffraction peak mirror pairs likewise rule out broken mirror symmetry. Since low-energy electrons undergo multiple scattering due to their strong interaction with matter, electron diffraction intensities should not be compared directly to the kinematic calculation results. Nevertheless, the symmetry of the surface should still be reflected in the symmetry of the electron diffraction pattern, regardless of the nature of the physical interaction between incident electrons and the solid surface. ${ }^{40}$ Furthermore, the range of incident energies that is considered here is at the minimum of the electron mean-free path in solids. ${ }^{40}$ This assures that the contribution of the graphene layer to the diffraction signal is maximized. Therefore the absence of broken mirror symmetry in $\mu$ LEED measurements indicates either that $\mathrm{g} / \mathrm{Ru}(0001)$ is racemic on a length scale of $250 \mathrm{~nm}$ or that it is possibly not even chiral.

\section{Small-angle lattice rotations}

An interesting observation that was made in the course of the $\mu$ LEED measurements is that the graphene-derived fractional-order satellite diffraction spots are seldom aligned exactly with the substrate reciprocal lattice. Instead, the entire ensemble of satellite spots around each $\mathrm{Ru}$ integer order diffraction spot is rotated as a group about their respective stationary foci. When the incident $\mu$ LEED beam is scanned across the surface, the rotation angle $\theta$ undulates randomly about a mean, $\theta=0$, that is aligned optimally with the substrate high-symmetry direction. ${ }^{41}$ Figure 3 (a) shows the synchronized rotations of the satellites about the $(0,0)$ and
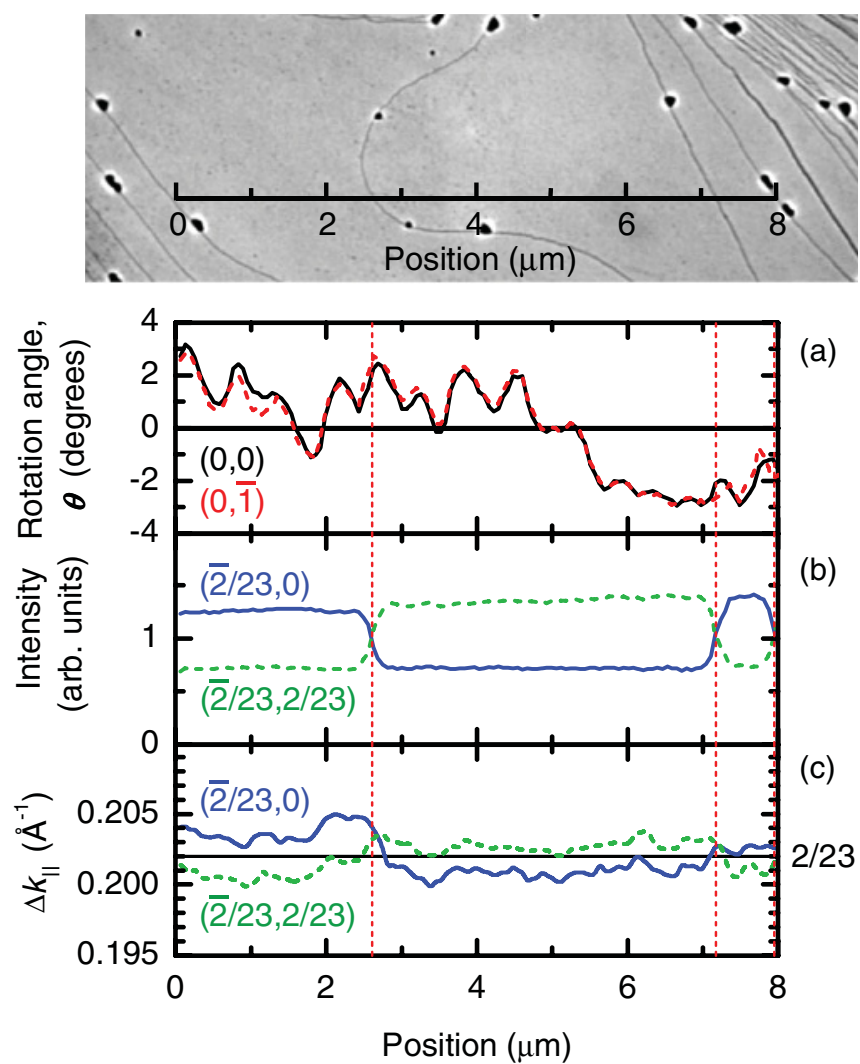

FIG. 3. (Color online) (a) The rotation angle $\theta$ of the graphenederived diffraction satellites about the $(0,0)$ (black, solid curve) and $(0, \overline{1})$ (red, dashed curve) spots from orientational coincidence with the substrate high-symmetry direction is plotted as a function of $\mu$ LEED beam position along the line indicated in the LEEM image at the top (imaging energy $E=20 \mathrm{eV}$ ). The variations of the (b) intensities and (c) parallel momentum transfers, $\Delta k_{\|}$, of the $(\overline{2} / 23,2 / 23)$ (green, dashed curves) and $(\overline{2} / 23,0)$ (blue, solid curves) spots are shown as a function of $\mu$ LEED beam position along the same line. Vertical dashed (red) lines indicate atomic step positions. The incident electron energy in (a)-(c) is $E=50 \mathrm{eV}$. 
$(0, \overline{1})$ spots that occur as the $\mu$ LEED beam is scanned along the line indicated in the accompanying LEEM image, also shown in the supplementary material. ${ }^{41}$ In this bright-field (BF) image, formed using the backscattered (00) diffraction beam, atomic steps are identified by dark lines that are produced by a phase contrast mechanism. ${ }^{42,43}$ Although a small amount of second-layer graphene appears as black dots in this example, similar rotations are observed when the second-layer graphene is absent.

The intensities of the $(\overline{2} / 23,2 / 23)$ and $(\overline{2} / 23,0)$ diffraction spots are also plotted as a function of $\mu$ LEED beam position in Fig. 3(b). The difference of these spot intensities reflects the threefold rotational symmetry of the surface termination on each terrace. Furthermore, the relative intensities of the two spots invert when the $\mu$ LEED beam traverses a single atomic step between terraces with rotated termination. This inversion is responsible for contrast in DF images (Fig. 2). Taking into account the angle between the scan direction and the step edge, the width of the intensity transition across a step independently confirms that the $\mu$ LEED beam size is below $250 \mathrm{~nm}$. Furthermore, the intensity inversion identifies atomic step positions accurately in diffraction mode. This information can be correlated directly with other structural characteristics that are detected by diffraction at the same time, e.g., Figs. 3(a) and $3(\mathrm{c})$.

DF imaging provides a confirmation and a two-dimensional representation of the rotations in this system. Although sharp superstructure diffraction spots are observed with $\mu$ LEED for a small illuminated area, diffraction spots are very slightly broadened tangentially (but remain sharp radially) when a larger area of the surface is illuminated, as for LEEM imaging. The broadening is produced by the superposition of sharp diffraction peaks with different rotations that are generated at different positions on the surface. DF images that are formed by concentric positioning of the contrast aperture on the superstructure diffraction spot are dominated by the contrast between terraces that reflects their different terminations [Figs. 2(a) and 2(b)]. However, when a DF image is formed from intensity predominantly in one half of the diffraction spot, upon a relative tangential displacement of the aperture and spot, additional contrast arises between areas within each terrace [Figs. 2(c)-2(f)]. We call this shadow dark-field (SDF) imaging. SDF contrast identifies regions in $\mathrm{g} / \mathrm{Ru}(0001)$ that produce oppositely rotated superstructure diffraction features. The contrast reversal that is observed in SDF images formed using intensity in oppositely rotated halves of the diffraction spots, labeled $(+)$ and $(-)$ in Fig. 2, confirms this interpretation [Figs. 2(c), 2(e) and Figs. 2(d), 2(f)]. The complementary SDF images in Fig. 2 reveal rotation domains with length scales similar to features observed in the one-dimensional $\mu$ LEED line scan [Fig. 3(a)], but also a great deal of fine structure below the $250-\mathrm{nm}$ lateral resolution of $\mu$ LEED.

The rotation that is evident in diffraction and SDF imaging here may arise from a rotation of the graphene with respect to the $\mathrm{Ru}$ lattice. This mutual rotation is accompanied by a larger rotation of the moiré pattern in the superposition of the two lattices. Kinematic calculations show that rotation of the graphene lattice produces synchronized rotations of the satellite spots about each integer order spot as observed here [Fig. 2(a)]. The diffraction and moiré rotation angles are

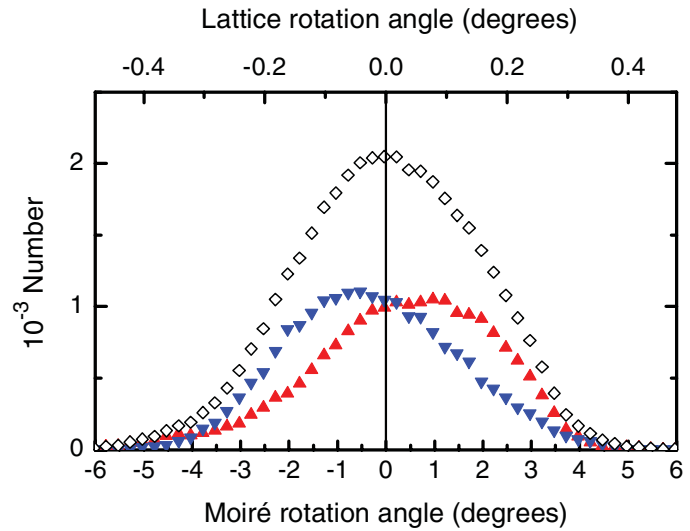

FIG. 4. (Color online) Normalized distributions of moiré (diffraction) and graphene lattice rotation angles are shown for the two surface terminations, labeled A $(\boldsymbol{\Lambda})$ and B $(\boldsymbol{\nabla})$, and composite $(\diamond)$ for both terminations.

equal. The moiré and lattice rotation angles are related by the periodicities of the two generating lattices. ${ }^{14}$ The angular magnification factor between moiré and lattice rotations is 12.5 for 25 -on-23 $\mathrm{g} / \mathrm{Ru}(0001)$.

The distribution of moiré rotation angles exhibited by $\mathrm{g} / \mathrm{Ru}(0001)$ was obtained by performing $\mu$ LEED measurements in two-dimensional (2D) scan arrays over large terraces on separately prepared graphene layers. The normalized distributions for the two different surface terminations, identified as $\mathrm{A}$ and $\mathrm{B}$ in Fig. 4, represent measurements at 20000 independent positions separated by the $\mu$ LEED beam size. These distributions are normalized by the number of data points that populate the data set, $\mathrm{A}: \mathrm{B} \approx 1.6: 1$. The distributions are clearly asymmetric, with peaks that are shifted oppositely from optimal alignment with the substrate high-symmetry direction, $\theta=0$. Thus the lattice rotation is biased by the surface termination.

Numerous rotational variants of graphene are known to form on many close-packed metal surfaces. ${ }^{4,9,12-17}$ Variants with large-angle lattice rotations ranging from several up to $30^{\circ}$ from the aligned "R0" orientation have been reported. Unlike the behavior on other metals, however, graphene has been reported to be present only in a single orientation on $\mathrm{Ru}(0001)$. $^{1,3,11,23}$ The relatively stronger interaction of graphene with the $\mathrm{Ru}$ surface compared with other metal surfaces ${ }^{1,9,11,15}$ may be a contributing factor in this high degree of orientational order. Nevertheless, the present investigations reveal a distribution of small-angle rotations about the nominal orientation in $\mathrm{g} / \mathrm{Ru}(0001)$. A similarly small, $\sim 0.5^{\circ}$, lattice rotation on $\mathrm{Ru}(0001)$ was also reported recently. ${ }^{26}$ Interestingly, small-angle rotations with a comparable angular spread have been observed in the R0 variant on $\operatorname{Ir}(111){ }^{14}$ which is among those more weakly interacting systems that exhibit a number of large-angle rotational variants. ${ }^{9,12,15}$ The present results therefore suggest that such small-angle lattice rotations could be the rule rather than the exception on metal surfaces.

The LEEM and $\mu$ LEED measurements do not provide direct insight about the lattice deformations or grain boundaries introduced by the presence of small-angle rotation domains. A grain boundary between two domains in graphene with 
different crystallographic orientation is generally comprised of a linear array of edge dislocations, the lowest energy configurations of which consist of bound pairs of complementary pentagon and heptagon disclinations. ${ }^{18,19}$ The ideally periodic spacing between these dislocations is dictated by the tilt angle across the boundary, with large dislocation spacings corresponding to the small-angle grain boundaries observed here. Hexagonal graphene units that remain intact along the linear boundary are naturally deformed slightly. Additionally, buckling further reduces the in-plane elastic energy of a dislocation at the cost of bending energy when graphene is not constrained to a planar geometry. ${ }^{18}$ This energy minimization mechanism is especially important for small-angle grain boundaries. Although some details of the dislocation structure and energetics may differ for corrugated $\mathrm{g} / \mathrm{Ru}(0001)$, the key features discussed here are expected to be generally robust. The effects of defect structures at grain boundaries on electronic structure are pronounced. ${ }^{18-20}$ Crystal momentum conservation also determines the transmission characteristics of charge carriers across grain boundaries in graphene. ${ }^{19}$ This gives rise to boundaries that are either transmitting at all energies or perfectly reflecting at the Fermi energy within a transmission gap that is inversely proportional to the periodic distance separating dislocations at the boundary. ${ }^{19}$ Although the transmission gap will be small for the small-angle grain boundaries observed here, the proliferation of such boundaries could have a large accumulated effect on transport or other electronic properties.

\section{Unit-cell dimension and distortion}

Finally, we remark on the possibility that changes in graphene lattice spacing accompany lattice rotation. ${ }^{26}$ The half-width at half maximum of the instrumentally-limited $\mu$ LEED peak, $\Delta k_{\mu}=2.1 \times 10^{-2} \AA^{-1}$, precludes resolution of closely spaced peaks for 12-on-11, 25-on-23, and 13-on-12 periodicities, $\Delta k_{\|}=0.2112 \AA^{-1}(1 / 11), 0.202 \AA^{-1}(2 / 23)$, $0.1936 \AA^{-1}(1 / 12)$, respectively. However, relative changes of the diffraction peak position that could conceivably occur when the $\mu$ LEED beam alternately illuminates different rotation domains as it is scanned across the surface can be determined precisely. Although variations of $\Delta k_{\|}$for the $(\overline{2} / 23,2 / 23)$ and $(\overline{2} / 23,0)$ diffraction spots are observed when the $\mu$ LEED beam is scanned [Fig. 3(c)], they are insignificant compared to the variations that would be caused by the different lateral periodicities. Thus, lattice rotation cannot be invoked to explain the 12-on-11 or other deviation from 25 -on-23 periodicity. Interestingly, we find that $\Delta k_{\|}$ for these two diffraction peaks differs on average by $\sim 2 \times$ $10^{-3} \AA^{-1}$ [Fig. 3(d)]. Furthermore, the relative magnitudes of the respective $\Delta k_{\|}$invert when the $\mu$ LEED beam traverses a single atomic step. This observation implies the presence of a small biaxial distortion of the unit cell that is coupled with the surface termination. This surprising structural feature will not be so plainly detectable in measurements made using laterally averaging techniques that integrate over both surface terminations. The lateral resolution of STM may also not be sufficient to detect the small distortion reported here.

\section{CONCLUSION}

In conclusion, we find no evidence of chirality in $\mathrm{g} / \mathrm{Ru}(0001)$ comprising rotated features within the unit cell as proposed in Ref. 28 based on SXRD and elastic energy minimization arguments. This means that $\mathrm{g} / \mathrm{Ru}(0001)$ is already racemic on the short 250-nm length scale of the $\mu$ LEED measurement carried out in these investigations. Knowledge of this racemic length scale is crucial for future characterization of the potential novel properties associated with chirality. Alternatively, the possibility remains that $\mathrm{g} / \mathrm{Ru}(0001)$ is not chiral at all. The observation that small-angle lattice rotations are present on a longer length scale that are biased by the substrate termination suggests energy minimization is achieved by slightly rotating the entire graphene layer. The prevalence of small-angle lattice rotation domains also implies the presence of small-angle grain boundaries. Although the defect density associated with a single small-angle boundary may be low, the proliferation of such boundaries implies a large defect density, whose accumulated effect on the physical properties of the layer may be significant. These results confirm that the orientational uniformity of graphene grown on a $\mathrm{Ru}(0001)$ surface is not so perfect as previously thought, at least under the growth conditions that were optimized and employed here. Similar investigations of graphene on other metal surfaces, including those that exhibit large-angle rotational variants, and for various preparation methods could enlighten us about the full extent of the small-angle orientational disorder observed here.

\section{ACKNOWLEDGMENTS}

We are grateful to T. Greber, P. R. Willmott, A. Seitsonen, and $\mathrm{H}$. Brune for valuable discussions. *phaltman@ust.hk

${ }^{1}$ P.W. Sutter, J.-I. Flege, and E. A. Sutter, Nature Mater. 7, 406 (2008).

${ }^{2}$ X. Li, W. Cai, J. An, S. Kim, J. Nah, D. Yang, R. Piner, A. Velamakanni, I. Jung, E. Tutuc, S. K. Banerjee, L. Colombo, and R. S. Ruoff, Science 324, 1312 (2009).

${ }^{3}$ Y. Pan, H. Zhang, D. Shi, J. Sun, S. Du, F. Liu, and H. J. Gao, Adv. Mater. 21, 2777 (2009).

${ }^{4}$ R. Van Gastel, A. T. N'Diaye, D. Wall, J. Coraux, C. Busse, N. M. Buckanie, F.-J. Meyer zu Heringdorf, M. Horn von Hoegen, T. Michely, and B. Poelsema, Appl. Phys. Lett. 95, 121901 (2009).
${ }^{5}$ M. Xu, D. Fujita, K. Sagisaka, E. Watanabe, and N. Hanagata, ACS Nano 5, 1522 (2011)

${ }^{6}$ A. K. Geim and K. S. Novoselov, Nature Mater. 6, 183 (2007).

${ }^{7}$ C. Oshima and A. Nagashima, J. Phys.: Condens. Matter 9, 1 (1997).

${ }^{8}$ G. Giovannetti, P. A. Khomyakov, G. Brocks, V. M. Karpan, J. van den Brink, and P. J. Kelly, Phys. Rev. Lett. 101, 026803 (2008).

${ }^{9}$ J. Wintterlin and M.-L. Bocquet, Surf. Sci. 603, 1841 (2009).

${ }^{10}$ A. Allard and L. Wirtz, Nano Lett. 10, 4335 (2010).

${ }^{11}$ M. Gao, Y. Pan, C. Zhang, H. Hu, R. Yang, H. Lu, J. Cai, S. Du, F. Liu, and H. J. Gao, Appl. Phys. Lett. 96, 053109 (2010). 
${ }^{12}$ E. Starodub, A. Bostwick, L. Moreschini, S. Nie, F. El Gabaly, K. F. McCarty, and E. Rotenberg, Phys. Rev. B 83, 125428 (2011).

${ }^{13}$ Y. Murata, E. Starodub, B. B. Kappes, C. V. Ciobanu, N. C. Bartelt, K. F. McCarty, and S. Kodambaka, Appl. Phys. Lett. 97, 143114 (2010).

${ }^{14}$ A. T. N'Diaye, J. Coraux, T. N. Plasa, C. Busse, and T. Michely, New J. Phys. 10, 043033 (2008).

${ }^{15}$ P. Sutter, J. T. Sadowski, and E. Sutter, Phys. Rev. B 80, 245411 (2009).

${ }^{16}$ J. Coraux, A. T. N'Diaye, C. Busse, and T. Michely, Nano Lett. 8, 565 (2008).

${ }^{17}$ K. Kim, Z. Lee, W. Regan, C. Kisielowski, M. F. Crommie, and A. Zettl, ACS Nano 5, 2142 (2011).

${ }^{18}$ O. V. Yazyev and S. G. Louie, Phys. Rev. B 81, 195420 (2010)

${ }^{19}$ O. V. Yazyev and S. G. Louie, Nature Mater. 9, 806 (2010).

${ }^{20}$ J. Lahiri, Y. Lin, P. Bozkurt, I. I. Oleynik, and M. Batzill, Nat. Nanotechnol. 5, 326 (2010).

${ }^{21}$ P. M. Ajayan and B. I. Yakobson, Nature Mater. 10, 415 (2011).

${ }^{22}$ Q. Yu, L. A. Jauregui, W. Wu, R. Colby, J. Tian, Z. Su, H. Cao, Z. Liu, D. Pandey, D. Wei, T. F. Chung, P. Peng, N. P. Guisinger, E. A. Stach, J. Bao, S. S. Pei, and Y. P. Chen, Nat. Mater. 10, 443 (2011).

${ }^{23}$ S. Marchini, S. Günther, and J. Wintterlin, Phys. Rev. B 76, 075429 (2007).

${ }^{24}$ D. Martoccia, P. R. Willmott, T. Brugger, M. Björck, S. Günther, C. M. Schlepütz, A. Cervellino, S. A. Pauli, B. D. Patterson, S. Marchini, J. Wintterlin, W. Moritz, and T. Greber, Phys. Rev. Lett. 101, 126102 (2008).

${ }^{25}$ M.-C. Wu, Q. Xu, and D. W. Goodman, J. Phys. Chem. 98, 5104 (1994).

${ }^{26}$ B. Borca, S. Barja, M. Garnica, M. Minniti, A. Politano, J. M. Rodriguez-Garcia, J. J. Hinarejos, D. Farías, A. L. Vázquez de Parga, and R. Miranda, New J. Phys. 12, 093018 (2010).

${ }^{27}$ A. L. Vázquez de Parga, F. Calleja, B. Borca, M. C. G. Passeggi Jr., J. J. Hinarejos, F. Guinea, and R. Miranda, Phys. Rev. Lett. 100, 056807 (2008).
${ }^{28}$ D. Martoccia, M. Björck, C. M. Schlepütz, T. Brugger, S. A. Pauli, B. D. Patterson, T. Greber, and P. R. Willmott, New J. Phys. 12, 043028 (2010).

${ }^{29}$ W. Moritz, B. Wang, M.-L. Bocquet, T. Brugger, T. Greber, J. Wintterlin, and S. Günther, Phys. Rev. Lett. 104, 136102 (2010).

${ }^{30}$ T. Brugger, S. Günther, B. Wang, J. H. Dil, M.-L. Bocquet, J. Osterwalder, J. Wintterlin, and T. Greber, Phys. Rev. B 79, 045407 (2009).

${ }^{31}$ A. T. N'Diaye, T. Gerber, C. Busse, J. Mysliveček, J. Coraux, and T. Michely, New J. Phys. 11, 103045 (2009).

${ }^{32}$ M. Roos, D. Künzel, B. Uhl, H. H. Huang, O. B. Alves, H. E. Hoster, A. Gross, and R. J. Behm, J. Am. Chem. Soc. 133, 9208 (2011).

${ }^{33}$ M. Fujita, K. Wakabayashi, K. Nakada, and K. Kusakabe, J. Phys. Soc. Jpn. 65, 1920 (1996)

${ }^{34}$ Y. W. Son, M. L. Cohen, and S. G. Louie, Nature (London) 444, 347 (2006).

${ }^{35}$ E. Bauer, Rep. Prog. Phys. 57, 895 (1994).

${ }^{36}$ M. S. Altman, J. Phys.: Condens. Matter 22, 084017 (2010).

${ }^{37}$ G. E. Thayer, J. T. Sadowski, F. Meyer zu Heringdorf, T. Sakurai, and R. M. Tromp, Phys. Rev. Lett. 95, 256106 (2005).

${ }^{38}$ J. de la Figuera, J. M. Puerta, J. I. Cerda, F. El Gabaly, and K. F. McCarty, Surf. Sci. 600, L105 (2006).

${ }^{39} \mathrm{P}$. R. Willmott (private communication).

${ }^{40}$ M. A. Van Hove, W. H. Weinberg, and C. M. Chan, Low Energy Electron Diffraction (Springer Verlag, Berlin Heidelberg, 1986).

${ }^{41}$ See Supplemental Material at http://link.aps.org/supplemental/ 10.1103/PhysRevB.84.235415 for a video of the synchronized diffraction spot rotations summarized in Fig. 3.

${ }^{42}$ W. F. Chung and M. S. Altman, Ultramicroscopy 74, 237 (1998).

${ }^{43}$ A. B. Pang, Th. Müller, M. S. Altman, and E. Bauer, J. Phys.: Condens. Matter 21, 314006 (2009). 